\title{
Loss optimization in double fishnet metamaterials at telecommunication wavelengths
}

\section{S. Iyer \\ srsi@kth.se \\ S. Popov}

\section{A. T. Friberg}

\begin{abstract}
Institute of Technology (KTH), Department of Microelectronics and Applied Physics, Electrum 229, SE-164 40 Kista, Sweden

Institute of Technology (KTH), Department of Microelectronics and Applied Physics, Electrum 229, SE-164 40 Kista, Sweden

Institute of Technology (KTH), Department of Microelectronics and Applied Physics, Electrum 229, SE-164 40 Kista, Sweden

Department of Applied Physics, Aalto University, PO Box 13500, FI-00076 Aalto, Finland

Department of Physics and Mathematics, University of Eastern Finland, PO Box 111, FI-80101 Joensuu, Finland
\end{abstract}

A hole shape optimization study was made for a double fishnet metamaterial producing a negative index of refraction within $1.4-1.5 \mu \mathrm{m}$. It is found that within these wavelengths, elliptical holes offer lower losses as compared to rectangular ones and theoretically produce the best figure of merit (FOM) of approximately 6. [DOI: 10.2971/jeos.2011.11008]

Keywords: metamaterials, plasmonics, metal optics

\section{INTRODUCTION}

Metamaterials (MMs) with negative effective refractive index $\left(n_{e f f}\right)$, also known as left-handed metamaterials, has been a hot topic of research since the last decade. MMs possess physical properties that can be exploited in exotic applications like super lenses and optical cloaks just to name a few [1]. However, these special properties are not found in any naturally available material. So, it needs to be constructed artificially using fundamental components made of metal $(\mathrm{Au} / \mathrm{Ag})$ and dielectrics that provide resonant negative electrical and magnetic response. Noble metals seem to be the only choice for now in constructing MMs and losses are still a major impediment that prevent commercial realization of $\mathrm{MM}$ devices in the optical range. But, in the last two years, techniques combining MMs with gain media are being used to compensate for the intrinsic loss in metals [2, 3].

Among the various fundamental designs proposed for MMs (with no gain media) in the near-infrared (NIR) wavelength range, the best performance has been achieved using double metal layer fishnet structures (or double fishnet structures) $[1,4]$. These structures are made by sandwiching a transparent dielectric layer between two thin metal films and drilling holes periodically in a square array throughout the whole thickness. The most important aspect of the fishnet design is its ability to provide a negative refractive index over a small range of wavelengths even at normal incidence. This range of wavelength can be further increased by stacking several double fishnet structures on top of each other [5]. The parameter which defines the performance of any MM is the figure of merit (FOM). FOM is equal to the ratio $-n_{r} / n_{i}$, where $n_{r}$ and $n_{i}$ are the real and imaginary parts of the complex refractive index of the metamaterial. The imaginary part signifies the losses in the MM and hence, the FOM is desired to be as large as possible.

The different fishnet hole shapes considered until now are rectangular [6], circular [7] and elliptical [8] at various wavelengths. Our focus in this article is on improving the performance of a fundamental double fishnet structure operating in the vicinity of wavelengths $1.4-1.5 \mu \mathrm{m}$ where there is scope for some interesting telecommunication applications. The best value of FOM using rectangular holes achieved experimentally till date is $\sim 3$, at $1.4 \mu \mathrm{m}$ [6]. It has been found theoretically that within the telecommunication range, rectangular holes yield a larger value of FOM as compared to circular and square holes [7]. Introducing an aspect ratio in the hole is found to improve the transmission properties of the fishnet MM. Also, the effect of metal layer thickness $(t)$ on the FOM has been studied recently for the case of rectangular holes [9] and it has been confirmed by us that the FOM can be increased to $\sim 5$. However, we find that an even higher value of FOM $(\sim 6)$ can be obtained using elliptical holes. There is a lack of clarity in existing literature regarding what happens to the FOM and other effective MM parameters when the periodicity of the fishnet unit cell varies. Our article tries to address this rather practical issue in order to efficiently design a double fishnet MM at any given wavelength of interest. 


\section{OVERWIEW OF FISHNET STRUCTURES AND DEVELOPMENTS WITH RESPECT TO FOM}

A double fishnet MM structure can be logically split in two parts: electric and a magnetic quasi-atoms (or resonators), that needs to provide the required negative effective permittivity $\left(\varepsilon_{e f f}\right)$ and negative effective permeability $\left(\mu_{e f f}\right)$ respectively, at desired wavelengths. A one-dimensional array of thin long wires (oriented along polarization of the incident E-field) forms the electric atoms, where the wire parameters and period can be adjusted to tune the value of resulting plasma wavelength [10]. Another array of a pair of long metal strips (oriented along the incident $\mathrm{H}$-field) with dielectric between them form the magnetic atoms capable of providing a diamagnetic response ergo negative $\mu_{\text {eff }}$ [11]. The magnetic atom here is basically a simple LC circuit whose resonance condition depends on the geometrical dimensions of the metal (that defines inductance L) and dielectric (capacitance C). However, when a fishnet is fabricated, there is a coupling between both the resonators [12] and hence, the resulting values of $\varepsilon_{e f f}$ and $\mu_{e f f}$, are bound to be influenced by each other. The overall losses of the fishnet MM also significantly depend on the hole shape and size [7] in addition to the unavoidable intrinsic loss of the metal.

In 2006, Dolling et al. [6] fabricated a double fishnet structure with rectangular holes operating at $1.4 \mu \mathrm{m}$ wavelength with a FOM of $\sim 3$ and this has been the best experimental FOM value reported until today. However, the same group realized that the fabricated structure was not optimized properly for the given metal thickness and also, a comparison was made between optimized fishnets with rectangular, circular and square holes set to work at $1.4 \mu \mathrm{m}$ [7]. It was found that the FOM of rectangular holes $(\sim 3.7)$ was at least two times better than circular and square holes $(\sim 1.6)$. There are a number of geometrical parameters that need to be adjusted namely lattice constant $a$, hole dimensions $m$ and $n$, metal thickness $t$ and dielectric thickness $d$ (refer Figure 1) in order to get an optimum FOM for a given wavelength of operation. The dimensions $d$ and $t$ were then varied and the best theoretical FOM value possible for rectangular hole is $\sim 5$ (at $t=90 \mathrm{~nm}$, $d=60 \mathrm{~nm})$ at $1.4 \mu \mathrm{m}$ wavelength $[9,13]$. Fishnet structures with elliptical holes have been investigated in the past but at longer wavelengths; and it was found that elliptical holes provide almost the same FOM as rectangular holes [8]. However, this particular investigation was done before the influence of metal layer thickness on the FOM of a double fishnet MM was studied. Thus, we will try to compare the best possible theoretical performances of rectangular and elliptical holes within the $1.4-1.5 \mu \mathrm{m}$ window taking into account the issue of metal layer thickness in the forthcoming section.

\section{SIMULATION METHOD AND MODEL PARAMETERS}

The modelling is done using a commercial software CST MicroWave Studio, which is based on finite integration time domain approach. Figure 1 shows an example of the unit cell of a fishnet MM with elliptical holes and excitation con- ditions studied in this manuscript. The metal used is $\mathrm{Ag}$ since it offers lower losses at optical wavelengths as compared to $\mathrm{Au}[5,6]$. The dimensions $m$ and $n$ denote the diameter of major axis (length) and minor axis (breadth) for elliptical (rectangular) holes. The dispersion parameters for $\mathrm{Ag}$ is set as follows: free electron Drude model with plasma frequency $\omega_{p}=1.37 \times 10^{16} \mathrm{~s}^{-1}$ and scattering frequency $v=8.5 \times 10^{13} \mathrm{~s}^{-1}$. The refractive index of dielectric $\mathrm{MgF}_{2}$ is 1.38. In our analysis, the whole structure is embedded in an effective homogeneous medium of refractive index 1.05 so as to render the system reciprocal. The modelling procedure is kept consistent with Refs. [6, 7, 9]. The effective parameters $\left(n_{e f f}, \varepsilon_{e f f}\right.$ and $\left.\mu_{e f f}\right)$ of the double fishnet MM are retrieved from the complex transmitted and reflected fields using the standard parameter extraction method [14, 15]. FOM, as mentioned before, is the ratio of real and imaginary parts of $n_{e f f}$ (i.e., $\left.\mathrm{FOM}=-n_{r} / n_{i}\right)$. The lattice constant $a$ and $\mathrm{MgF}_{2}$ thickness $d$ is set to $600 \mathrm{~nm}$ and $60 \mathrm{~nm}$ respectively.

\section{RESULTS AND DISCUSSION}

We first try to adjust the values of $m$ and $n$ for elliptical holes in order to get the best possible FOM within $1.4-1.5 \mu \mathrm{m}$ for $t=30 \mathrm{~nm}$ to $130 \mathrm{~nm}$. Physically, it is the dimension $w_{e}$ (or $m$ ) that mainly determines the magnitude of negative $\varepsilon_{e f f}$, and
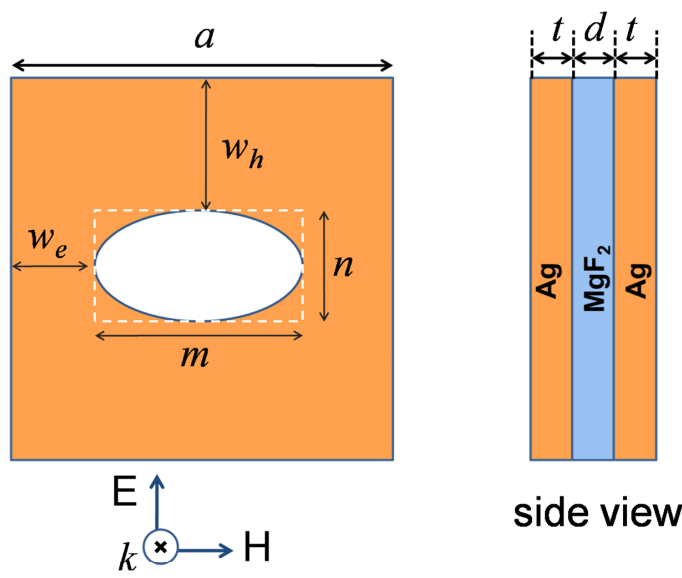

FIG. 1 The top and side view of the unit cell of double fishnet metamaterial.

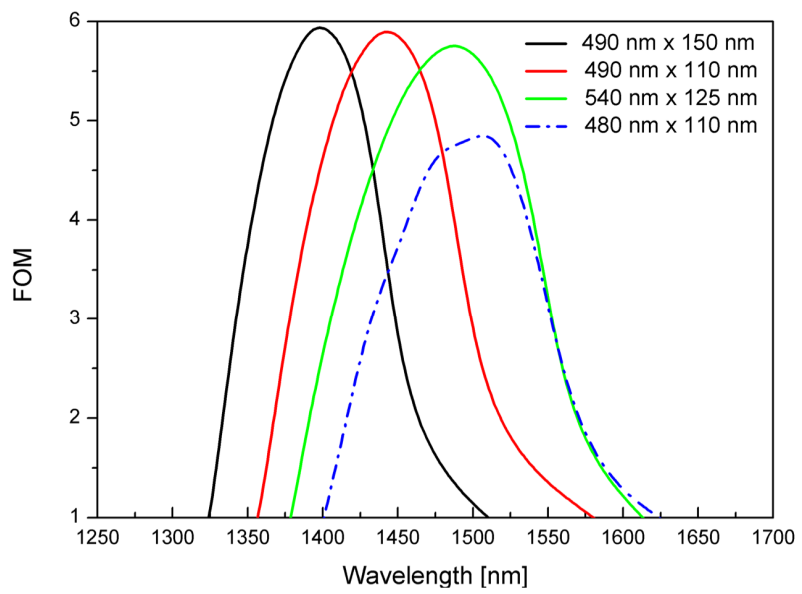

FIG. 2 Optimized elliptical holes $(m \times n)$ tuned to give the best FOM at 1.4, 1.45 and $1.5 \mu \mathrm{m}$, and rectangular hole (dot-dash blue) at $1.5 \mu \mathrm{m}$. 

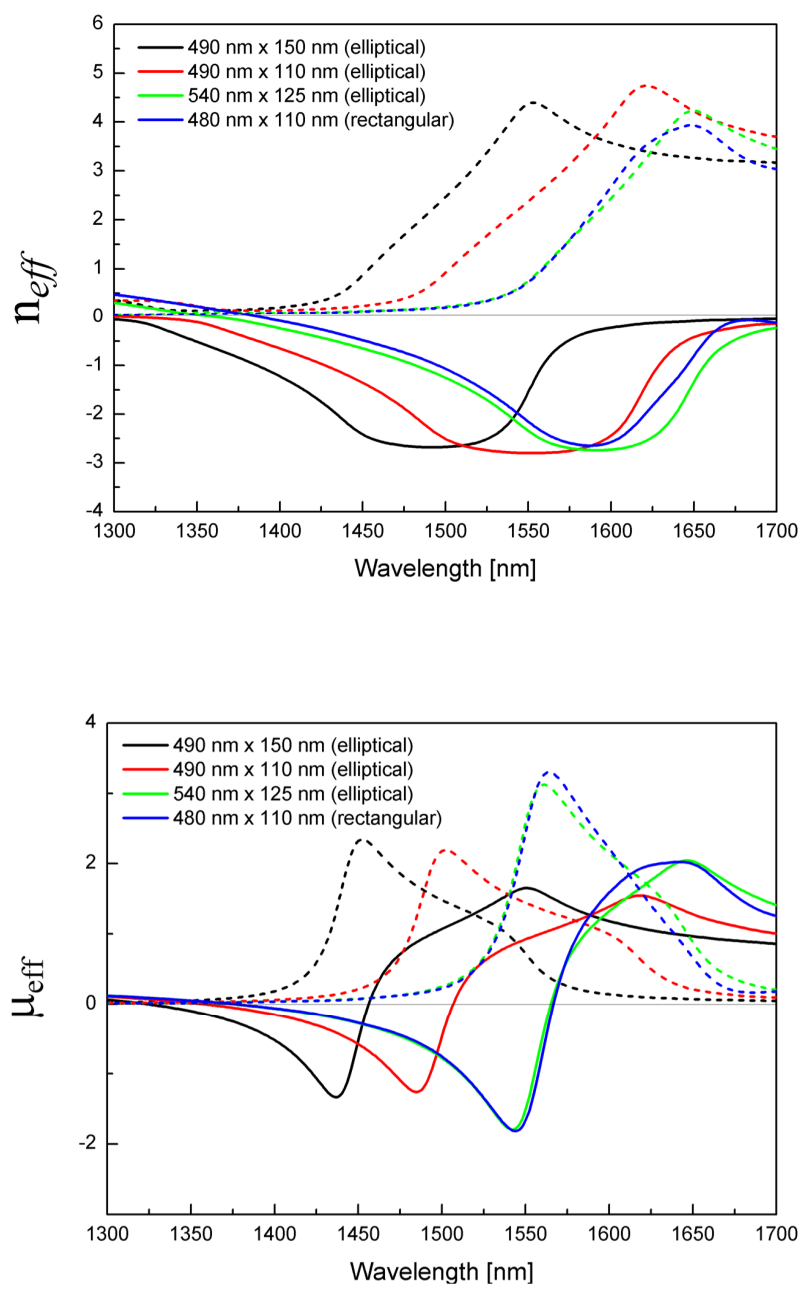

$w_{h}$ (or $n$ ) that controls the resonance wavelength of magnetic atoms. It is found that the max. FOM of elliptical holes is almost similar to that of rectangular holes for most values of $t$. The max. FOM values possible for rectangular holes as a function of $t$ is given in Ref. [9]. However, at $t=90 \mathrm{~nm}$, we see an improvement in the FOM reaching approximately 6. As mentioned before, the best possible FOM for rectangular holes at $t=90 \mathrm{~nm}$ is $\sim 5$. By carefully adjusting $m$ and $n$ (indirectly $w_{e}$ and $\left.w_{h}\right)$, it is possible to tune the operating point of the double fishnet MM without much degradation in the FOM peak value as shown in Figure 2.

Though the improvement is not very drastic, it is still quite significant highlighting the importance of hole shape in engineering the losses of a metamaterial structure. The average transmission from optimized elliptical holes within $1.4-1.5 \mu \mathrm{m}$ is $\sim 70 \%$ (not shown) which is also greater than that of rectangular holes $(\sim 60 \%)$. The dependence of losses (or FOM) on the hole shape is vaguely related to the holewaveguide resonance and the dielectric gap surface plasmon modes but the exact explanation is still elusive [16]. Figure 3 shows the extracted effective parameters corresponding to the FOM curves in Figure 2. It can be seen that the FOM improvement for elliptical holes is predominantly due to slightly higher magnitude of $n_{r}$.

Next, we check the effect of small variations in the lattice constant $a$ on the double fishnet MM parameters keeping the hole

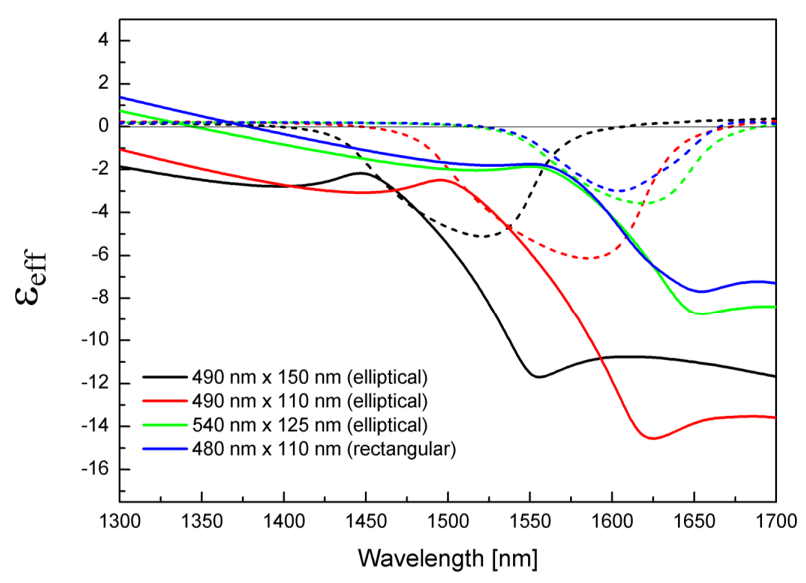

FIC. 3 The effective parameters (a) $n_{e f f}$, (b) $\mu_{e f f}$ and (c) $\varepsilon_{e f f}$ retrieved from the complex transmitted and reflected fields for the optimized fishnet structures. The solid lines represent the real part and dashed lines represent the imaginary part of the respective parameters. The colour notation (legends) is the same as in Figure 2.

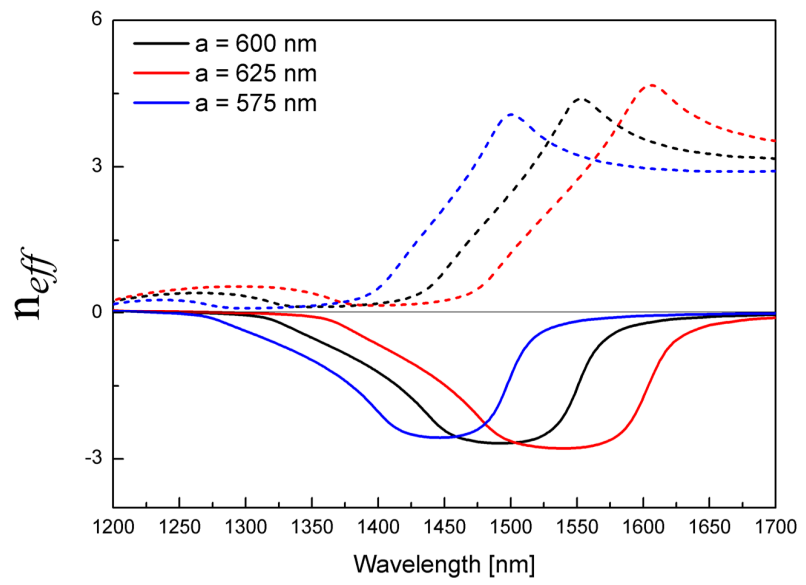

FIC. 4a Comparison of effective parameters (a) $n_{\text {eff }}$, (b) $\mu_{\text {eff }}$, (c) $\varepsilon_{e f f}$ and (d) FOM corresponding to an elliptical hole $(490 \mathrm{~nm} \times 150 \mathrm{~nm})$ for small variations in unit cell period $a$.

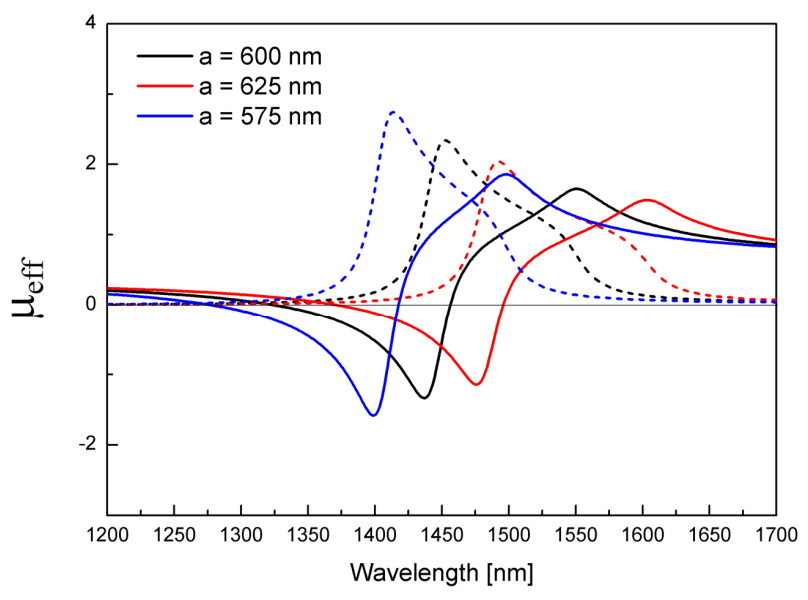

FIG. 4 b Comparison of effective parameters (a) $n_{e f f}$, (b) $\mu_{e f f}$, (c) $\varepsilon_{e f f}$ and (d) FOM corresponding to an elliptical hole $(490 \mathrm{~nm} \times 150 \mathrm{~nm})$ for small variations in unit cell period $a$.

dimensions fixed. The case of an elliptical hole $(m=490 \mathrm{~nm}$, 


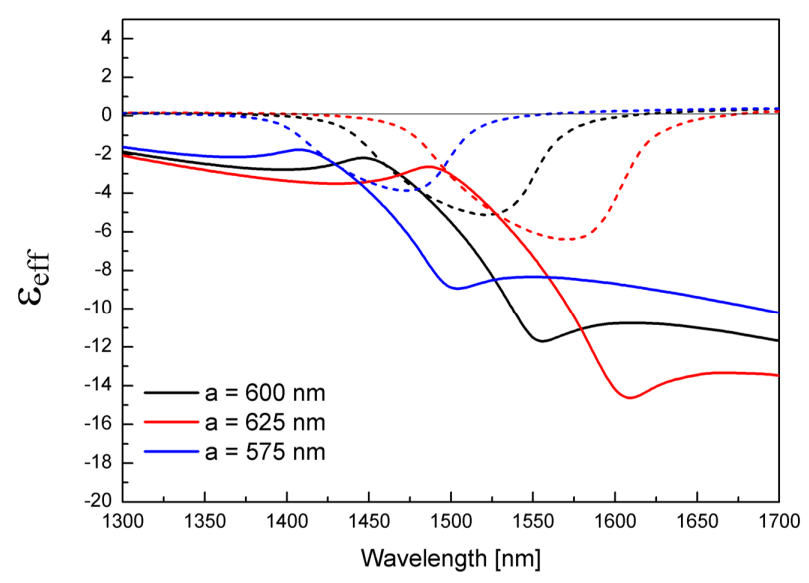

FIC. 4C Comparison of effective parameters (a) $n_{e f f}$, (b) $\mu_{e f f}$, (c) $\varepsilon_{e f f}$ and (d) FOM corresponding to an elliptical hole $(490 \mathrm{~nm} \times 150 \mathrm{~nm})$ for small variations in unit cell period $a$.

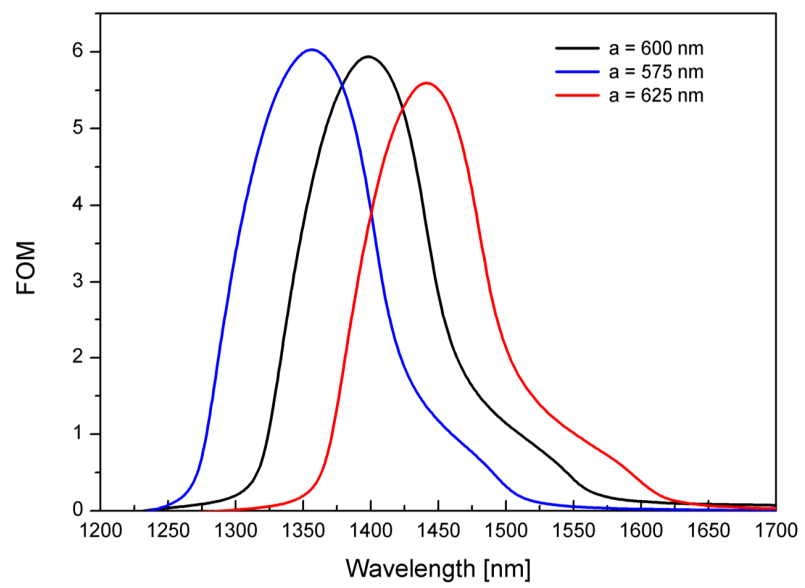

FIC. 4d Comparison of effective parameters (a) $n_{e f f}$, (b) $\mu_{\text {eff }}$, (c) $\varepsilon_{e f f}$ and (d) FOM corresponding to an elliptical hole $(490 \mathrm{~nm} \times 150 \mathrm{~nm})$ for small variations in unit cell period $a$.

$n=150 \mathrm{~nm}$ ) that gives maximum FOM at $1.4 \mu \mathrm{m}$ is considered. The effective MM parameters are calculated for $a=575 \mathrm{~nm}$, $600 \mathrm{~nm}$ and $625 \mathrm{~nm}$ respectively (Figure 4). A change in the periodicity alters both, $w_{e}$ and $w_{h}$. Hence, the response of electric and magnetic atoms, as well as the coupling conditions between them is also altered. It can be observed that there is a steady blue shift in the magnetic resonance $\left(\mu_{e f f}\right)$ accompanied by an increase in its magnitude as $a$ is decreased (Figure 4(b)). The blue shift is due to a change in the LC-resonance condition of the magnetic atom: a narrow $w_{h}$ corresponds to a smaller resonance wavelength. The increase in magnitude can be explained qualitatively by the larger density of magnetic atoms. The absolute value of effective permittivity decreases with $a$ (or $w_{e}$ ) partially because of reduced screening by the metal wires parallel to the incident E-field (Figure 4(c)). The stability of FOM peak value $(\sim 6)$ at the three different periodicities is the result of a coupling process between the electric and magnetic atoms (Figure 4(d)) as the decrease in permittivity is compensated by an increase in permeability. However, there is a gradual degradation in FOM peak as $a<575 \mathrm{~nm}$ and $a>625 \mathrm{~nm}$.

\section{SUMMARY}

A comparison was made between the best possible FOM and other effective optical parameters achieved by a double fishnet metamaterial with rectangular and elliptical holes designed to operate at telecommunication wavelengths. Elliptical holes ( $\max \mathrm{FOM}=\sim 6$ ) offer a better performance than rectangular holes $(\sim 5)$ within wavelengths $1.4-1.5 \mu \mathrm{m}$. A change in lattice periodicity influences the electric as well as the magnetic atoms of the MM and it could be an important parameter for optimization of a fishnet design.

\section{ACKNOWLEDGEMENTS}

This work was financially supported by the Academy of Finland (SA).

\section{References}

[1] V. M. Shalaev, "Optical negative-index metamaterials" Nat. Photonics 1, 41-48 (2007).

[2] E. Plum, V. A. Fedotov, P. Kuo, D. P. Tsai, and N. I. Zheludev, "Towards the lasing spaser: controlling metamaterial optical response with semiconductor quantum dots" 0pt. Express 17, 8548-8551 (2009).

[3] S. Xiao, V. P. Drachev, A. V. Kildishev, X. Ni, U. K. Chettiar, H.-K. Yuan and V. M. Shalaev, "Loss-free and active optical negative-index metamaterials" Nature 466, 735 (2010).

[4] J. Valentine, S. Zhang, T. Zentgraf, E. Ulin-Avila, D. A. Genov, G. Bartal, and X. Zhang, "Three-dimensional optical metamaterial with a negative refractive index" Nature 455, 376-380 (2008).

[5] S. Zhang, W. Fan, N. C. Panoiu, K. J. Malloy, R. M. Osgood, and S. R. Brueck, "Optical negative-index bulk metamaterials consisting of $2 \mathrm{D}$ perforated metal- dielectric stacks" Opt. Express 14, 6778-6787 (2006).

[6] G. Dolling, C. Enkrich, M. Wegener, C. M. Soukoulis, and S. Linden, "Low-loss negative-index metamaterial at telecommunication wavelengths" Opt. Lett. 31, 1800-1802 (2006).

[7] G. Dolling, M. Wegener, C. M. Soukoulis, and S. Linden, "Designrelated losses of double-fishnet negative-index photonic metamaterials" Opt. Express 15, 11536-11541 (2007).

[8] S. Zhang, W. Fan, K. J. Malloy, S. R. J. Brueck, N. C. Panoiu, and R. M. Osgood, "Demonstration of metal-dielectric negative-index metamaterials with improved performance at optical frequencies" J. Opt. Soc. Am. B 23, 434-438 (2006).

[9] P. Ding, E. J. Liang, Q. Zhou, L. Zhang, Y. X. Yuan, and Q. Z. Xue , "Influence of metal-layer thickness on the losses in fishnet metamaterial" in Proceedings to the International Workshop on Metamaterials, 191-193 (IEEE, Nanjing, 2008).

[10] J. B. Pendry, A. J. Holden, D. J. Robbins, and W. J. Stewart, "Low frequency plasmons in thin wire structures" J. Phys.-Condens. Mat. 10 (22), 4785-4809 (1998).

[11] V. A. Podolskiy, A. K. Sarychev, and V. M. Shalaev, "Plasmon modes in metal nanowires and left-handed materials" J. Nonlinear 0pt. Phys. 11, 65-74 (2002).

[12] S. Zhang, W. Fan, K. J. Malloy, S. R. Brueck, N. C. Panoiu, and R. M. Osgood, "Near-infrared double negative metamaterials" 0 pt. Express 13, 4922-4930 (2005). 
[13] J. Zhou, T. Koschny, and C. M. Soukoulis, "An efficient way to reduce losses of left-handed metamaterials" Opt. Express 16, 11147-11152 (2008).

[14] D. R. Smith, S. Schultz, P. Markos, and C. M. Soukoulis, "Determination of effective permittivity and permeability of metamaterials from reflection and transmission coefficients" Phys. Rev. B 65, 195104 (2002).

[15] X. Chen, T. M. Grzegorczyk, B. I. Wu, J. Pacheco, and J. A. Kong, "Robust method to retrieve the constitutive effective parameters of metamaterials" Phys. Rev. E Stat. Nonlin. Soft Matter Phys. 70, 016608 (2004).
[16] A. Mary, S. G. Rodrigo, F. J. Garcia-Vidal, and L. Martin-Moreno, "Theory of Negative-Refractive-Index Response of Double-Fishnet Structures" Phys. Rev. Lett. 86, 1114-1117 (2008). 